\title{
Carnets
}

Revue électronique d'études françaises de l'APEF

Deuxième série - 19 | 2020

Petite fabrique d'interprètes

\section{La musique du personnage}

\section{Vincent Jouve}

\section{(2) OpenEdition}

Journals

Édition électronique

URL : http://journals.openedition.org/carnets/11741

DOI : 10.4000/carnets. 11741

ISSN : 1646-7698

Éditeur

APEF

\section{Référence électronique}

Vincent Jouve, «La musique du personnage », Carnets [En ligne], Deuxième série - 19|2020, mis en ligne le 31 mai 2020, consulté le 23 décembre 2020. URL : http://journals.openedition.org/carnets/ 11741 ; DOI : https://doi.org/10.4000/carnets. 11741

Ce document a été généré automatiquement le 23 décembre 2020.

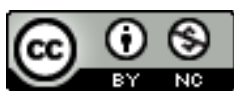

Carnets est mis à disposition selon les termes de la licence Creative Commons - Atribution - Pas d'utilisation commerciale 4.0 International. 


\title{
La musique du personnage
}

\author{
Vincent Jouve
}

1 Parler de «musique » à propos du personnage de roman peut sembler provocateur. La lecture est, à de rares exceptions près, une activité silencieuse et l'on peut légitimement se demander de quelle manière les figures issues du texte sont susceptibles de résonner. De fait, on a, jusqu'ici - du moins, à ma connaissance -, fort peu analysé le personnage romanesque en termes de sons. Si j'ai choisi de le faire aujourd'hui, c'est pour plusieurs raisons. La première tient à la dimension esthétique du roman. On se souvient que, selon Jakobson, la littérarité, «visée du message en tant que tel» (Jakobson, 1963: 218), est essentiellement travail sur le rythme et les sonorités, c'est-à-dire sur la musique. Pourquoi le personnage, élément fondamental de ce genre littéraire majeur qu'est le roman, ne serait-il pas concerné ? Les recherches sur la lecture ont, d'autre part, montré que le rapport au texte n'est pas seulement une aventure intellectuelle ou affective ; c'est aussi une expérience vécue par le corps. Cette dimension sensorielle de la lecture, qui tient au rythme du texte, à sa respiration, à la combinaison de sons qu'il met en place ${ }^{1}$, se retrouve dans la relation aux figures romanesques. Enfin, une étude de la musique des personnages a l'avantage de préciser leur sens et leur fonction dans un texte donné.

2 Mais que faut-il entendre par «musique du personnage »? L'expression est d'abord à comprendre au sens premier : il s'agit de l'impression sonore associée à un acteur du récit. On pourrait ainsi parler du "thème » d'un personnage de roman comme on parle du «thème » d'un personnage d'opéra. De même que, dans un film, l'arrivée d'un personnage est souvent accompagnée de musique, les figures d'un récit sont souvent liées à un entourage sonore qui permet de préciser leur valeur. Mais la musique du personnage peut également s'analyser sur le plan du signifié: il s'agira alors de s'interroger sur les relations qu'entretient tel acteur du roman avec le bruit en général et l'art musical en particulier.

3 Concrètement, comment procéder? A posteriori, quatre champs me semblent intéressants à analyser. Les deux premiers, le bruit du corps et le rapport à la musique, concernent le plan du contenu; les deux derniers, les métaphores musicales et le rythme $d u$ texte, sont à étudier au niveau de l'écriture. S'il est clair que ces quatre domaines 
sont particulièrement exploités dans les romans qui portent sur la musique, ils ont également une importance dans les autres récits. Je vais les examiner successivement en tentant de varier les exemples pour montrer la généralité du modèle.

\section{Le bruit du corps}

4 La réalité sonore d'un personnage, c'est d'abord celle qui nous est explicitement signalée par le texte. Sur le plan du signifié, le bruit du personnage vient d'abord de son corps et, en particulier, de sa voix. Cette dernière peut même prendre une importance fondamentale, comme dans ce passage d'Aurélien où le narrateur analyse la force de séduction de Bérénice :

La seule chose qu'il aima d'elle tout de suite, ce fut la voix. Une voix de contralto chaude, profonde, nocturne. Aussi mystérieuse que les yeux de biche sous cette chevelure d'institutrice. Bérénice parlait avec une certaine lenteur. Avec de brusques emballements, vite réprimés qu'accompagnaient des lueurs dans les yeux comme des feux d'onyx. (Aragon, 1966 : 33)

Pour Aurélien comme pour le lecteur, Bérénice est donc une musique avant d'être une image. Sa voix réconfortante et énigmatique, qui rassure et inquiète tout à la fois, fonctionne comme un thème musical. Dès sa première apparition, Bérénice résonne d'une musique complexe et ambivalente qui l'accompagnera jusqu'à la fin.

Que la voix soit l'une des composantes majeures de la musique du personnage est confirmé par la richesse de la palette musicale qui lui est associée. Le narrateur peut non seulement évoquer un timbre (sourd ou clair), mais aussi un ton (grave, autoritaire, suppliant, obséquieux). Il peut également mentionner un volume ou un débit. Voici comment, dans Les Misérables, sont rapportées les paroles de Champmathieu, homme fruste et naïf, traîné par erreur devant la justice :

Ce fut comme une éruption. Il sembla, à la façon dont les paroles s'échappaient de sa bouche, incohérentes, impétueuses, heurtées, pêle-mêle, qu'elles s'y pressaient toutes à la fois pour sortir en même temps. (Hugo, $1967: 300$ )

7 Le «bruit » du personnage, suite de sons à l'état brut qu'aucune opération artificielle ne parvient à mettre en musique, révèle ici la violence et la force de son indignation. Le primat du débit sur la construction syntaxique et l'impression de cacophonie qui en résulte témoignent de la spontanéité, de l'authenticité et, finalement, de l'innocence de Champmathieu.

8 Un texte peut aussi souligner une prononciation, voire, le cas échéant, un accent. On relèvera ainsi que, dans L $a$ Condition humaine, le personnage de Tchen "parl[e] français avec accentuation de gorge sur les mots d'une seule syllabe nasale» (Malraux, 1946: 61), particularité phonétique qui, évoquant un parler appuyé et monocorde, signale le fanatisme et l'intransigeance du terroriste.

La musique du corps, cependant, ne se limite pas à la voix. Il peut arriver, dans certains textes, que soient évoqués la légèreté ou la lourdeur d'un pas, le craquement des articulations ou le battement d'un pouls. Les bruits corporels ont d'ailleurs une valeur spécifique dont Bakhtine a montré la dimension carnavalesque ${ }^{2}$ : tout ce qui rappelle le corps en tant que tel nous renvoie au grotesque, au bas, au prosaïque par opposition à l'élevé, au poétique et au sublime. Si cette culture carnavalesque s'est particulièrement illustrée à la Renaissance, on la rencontre encore chez des écrivains comme Cohen. Bouffonne chez les Valeureux, la musique du corps évoque l'éclat et le défi s'agissant de 
Solal «referma[nt] les dents avec un claquement» (Cohen, 1958:60) au début du roman qui porte son nom.

10 Le bruit du corps, enfin, est parfois associé au froissement des habits. Ainsi, dans ce passage du Rivage des Syrtes, le vieux Danielo laisse entendre une musique assourdie, comme celle de l'autorité qu'il représente et qui ne parvient plus à se faire entendre comme elle le souhaiterait :

Il marchait très silencieux. Quand il se retournait, un léger bruissement de soie parcourait sa robe noire, et la flamme de la lampe oscillait faiblement. (Gracq, 1951: 306)

11 La musique du personnage, c'est donc d'abord le bruit naturel du corps. Mais ce dernier est parfois complété par des sonorités plus subtiles et travaillées, celles de l'art.

\section{Le rapport à la musique}

12 La dimension sonore d'une figure romanesque vient aussi de son rapport à la musique. Le lecteur associera spontanément le personnage à l'instrument dont il joue ou à la musique qu'il apprécie.

13 Le premier point va de soi. Il est clair que l'impression musicale produite par un personnage ne sera pas la même selon qu'on a affaire à un pianiste, un violoniste, un trompettiste ou un joueur de percussion. Dès les premières pages de L'Ecume des jours, Colin est ainsi associé à la musique qui sort de son pianocktail, cette fabuleuse machine qui compose des cocktails en fonction des airs que l'on joue. Les deux premiers morceaux évoqués étant Black and Tan Fantasy et Loveless Love, c'est au jazz, avec tout ce qu'il comporte d'expressivité et d'improvisation, voire de mélancolie, qu'est associé le personnage de Vian.

La qualité de l'impression musicale véhiculée par un personnage sera également différente (avec toutes les conséquences sur le plan de la signification) selon que le texte met en scène un simple amateur de musique, un professionnel, un prodige, voire un authentique créateur. L'écho d'un personnage sera d'autant plus net que sera faible la distance qui le sépare de la musique. Dans La Recherche, on entend plus facilement résonner la musique de Vinteuil (compositeur) que celle de Morel ou de Charlus (simples exécutants).

15 Enfin, il nous faudra distinguer, parmi les amateurs, entre ceux qui jouent d'un instrument, ceux qui préfèrent chanter ou siffloter et ceux qui se contentent d'écouter. Ainsi, dans Le Vice-consul de Duras, les trois personnages principaux se définissent par rapport à la musique: la mendiante "chante à tue-tête un chant enfantin de Battambang » (Duras, $1966: 28)$; Jean-Marc de H, le vice-consul, « sifflote Indiana's Song tout en marchant» (Duras, 1966: 35); quant à Anne-Marie Stretter, l'épouse de l'ambassadeur de France, elle, joue du piano avec, semble-t-il, un certain talent :

A Calcutta, presque chaque soir, elle joue. En passant sur le boulevard, on l'entend.

D'où qu'elle vienne, toutes en conviennent, elle a dû apprendre la musique très tôt,

à sept ans. A l'entendre, la musique serait ce qu'elle fait peut-être. (Duras, 1966 :

110-111)

16 La mendiante est donc associée aux sons primaires d'un chant archaïque et rudimentaire, derniers restes d'une enfance à laquelle elle est définitivement arrachée, Jean-Marc de $\mathrm{H}$ à un air léger et insouciant qui l'imprègne d'une certaine innocence et 
Anne-Marie Stretter à une musique noble, élégante, fragiles vestiges d'un passé heureux et perdu.

Grâce au langage, cependant, la petite musique du personnage ne vient pas seulement du bruit de son corps, de l'air qu'il apprécie ou de l'instrument qu'il joue, mais aussi des métaphores musicales ou sonores qui lui sont associées. Le plan du contenu est logiquement complété par le travail sur l'écriture.

\section{L'identité sonore}

18 On peut penser que si, comme j'en fais l'hypothèse, le personnage a bien, dans tout texte, une identité sonore, c'est essentiellement à travers les termes musicaux qui lui sont associés qu'elle se joue. Si le bruit du corps peut s'expliquer par certaines particularités physiques du personnage et son goût pour telle musique par des données culturelles ou sociales, les images musicales qui servent à le décrire ont une valeur plus générale : elles déterminent obliquement mais en profondeur la manière dont il est reçu par le lecteur.

19 Examinons, de ce point de vue, le début de Sodome et Gomorrhe : Charlus y est l'objet de deux analogies sonores. Il est d'abord associé à la musique de Beethoven :

(...) toutes les deux minutes, la même question semblait intensément posée à Jupien dans l'œillade de M. de Charlus, comme ces phrases interrogatives de Beethoven, répétées indéfiniment, à intervalles égaux, et destinées - avec un luxe exagéré de préparations - à amener un nouveau motif, un changement de ton, une « rentrée ». (Proust, 1989: 7)

Le baron, par la grâce de cette comparaison, est donc du côté de l'art, de la sensibilité, de la beauté. Mais, une page plus loin, il est associé à un son très différent, le bourdonnement d'un insecte :

$\mathrm{Au}$ même instant où $\mathrm{M}$. de Charlus avait passé la porte en sifflant comme un gros bourdon, un autre, un vrai celui-là, entrait dans la cour. (Proust, $1989: 8$ )

Charlus est donc également du côté de la nature, de l'instinct et du grotesque. Il incarne ainsi la double face de l'inverti, proche des créateurs par sa sensibilité exacerbée mais trop dépendant de ses désirs pour devenir un artiste accompli.

Plus près de nous, on se reportera au roman de Kundera, La Lenteur. Le narrateur y évoque en ces termes le membre de son personnage Vincent qui, après avoir connu un douloureux fiasco, se réveille soudainement:

(...) j'ai des doutes sur la raison de ce (...) membre qui, cette fois, a perdu tout bon sens ; sans aucun motif défendable, il se dresse contre l'univers comme la Neuvième Symphonie de Beethoven qui, face à la lugubre humanité, hurle son hymne à la joie. (Kundera, $1995:$ 163)

L'hymne à la joie, connotant, ici, la grandiloquence, fait particulièrement bien entendre le grotesque de la scène (l'érection, arrivant avec un temps de retard, est inopportune et déplacée) et le ridicule du personnage : Vincent, à l'image de la symphonie qui lui est accolée, apparaît stupidement démonstratif.

Une impression musicale peut parfois tenir au seul nom du personnage : elle lui est, alors, consubstantielle et l'accompagne d'un bout à l'autre du récit. C'est le cas d'olivier Cromorne, premier nom que Tournier envisagea pour le héros du Roi des Aulnes et qu'il donna par la suite à un personnage secondaire d'une de ses nouvelles. Si un tel nom est intéressant, c'est qu'il renvoie explicitement au domaine musical (le cromorne est un 
ancien instrument de musique à vent) et que, par son signifiant, il rattache la musique en question à la violence sauvage (croc) et à la mélancolie (morne) : on retrouve deux des caractéristiques de celui qui deviendra Abel Tiffauges, garagiste dépressif se métamorphosant en ogre ravisseur d'enfants.

Sur le plan de l'écriture, la musique du personnage ne se résume cependant pas à l'emploi de métaphores ou de comparaisons musicales; elle provient aussi - et peutêtre surtout - de la façon dont le personnage est mis en texte, c'est-à-dire des sonorités et des rythmes qui lui sont associés chaque fois qu'il apparaît dans le roman.

\section{L'être musical : de la mise en texte comme mise en musique}

Concernant la mise en texte, la musicalité du personnage peut d'abord s'inscrire dans les sonorités mêmes de son nom. Que l'on songe à "Tartarin de Tarascon ", nom qui laisse entendre les rodomontades du personnage haut en couleurs qui le porte, ou à " Rastignac ", âpre et acharné comme l'individu qu'il désigne, voire à "Raskolnikov ", aussi chaotique et bouillonnant que le héros auquel il renvoie. Dans un autre registre, on opposera chez Proust la légèreté des deux syllabes du nom «Elstir » à l'ampleur toute aristocratique du nom «Guermantes », indéfiniment prolongé par le «e » muet final.

Outre ces cas particuliers, c'est souvent lors de la première apparition d'un personnage que la dimension musicale et rythmique de son portrait apparait le plus nettement. Examinons le début de Toine, une nouvelle de Maupassant:

On le connaissait à dix lieues aux environs le père Toine, le gros Toine, Toine-maFine, Antoine Mâcheblé, dit Brûlot, le cabaretier de Tournevent. (Maupassant, 1974 :

Cette première évocation du héros éponyme nous le présente à travers un rythme syncopé et progressif, résultat d'une gradation qui traduit bien la vitalité et l'énormité du personnage. On retrouve ces caractéristiques quelques paragraphes plus loin dans le portrait proprement dit :

Ah! oui, on le connaissait Toine Brûlot, le plus gros homme du canton, et même de l'arrondissement. Sa petite maison semblait dérisoirement trop étroite et trop basse pour le contenir, et quand on le voyait debout sur sa porte où il passait des journées entières, on se demandait comment il pourrait entrer dans sa demeure. (Maupassant, 1974 : 427) Les répétitions ("trop étroite», "trop basse »), les allitérations en /r/ («Brûlot », " gros ", « arrondissement ", « dérisoirement », etc.), associent le personnage à la joie, au rire, à la force vitale : Toine semble présenté sur le fond sonore d'une chanson qui laisse entendre le même refrain tonique et gai.

Si dans les récits courts, la mise en musique d'un personnage reste relativement constante, il n'en va pas de même dans les récits plus longs et plus complexes. Dans un roman, l'identité rythmique d'un personnage n'est pas forcément la même à chacune de ses apparitions. Reportons-nous à ce passage de L'Ecume des jours qui fait entendre une musique lourde et pesante, celle qui envahit désormais la vie de Colin, accablé par le poids du destin :

(...) ses yeux ne voyaient plus que les laideurs des gens, sans cesse il annonçait les malheurs à venir ; sans cesse on le chassait, avec des coups, des cris, des larmes, des 
injures.

Il monta les deux marches, et suivit le couloir et frappa, reculant d'un pas sitôt après (...). (Vian, 1996 : 285)

La cadence toute mécanique des alexandrins blancs fait du personnage, à cet instant du récit, une sorte de robot qui n'a plus prise sur les événements.

L'évolution musicale d'un personnage n'est pas moins significative que son évolution psychologique ou actantielle. Anne Desbaresdes est, dans Moderato cantabile, un exemple intéressant de la façon dont un personnage peut se transformer «musicalement». Sa première apparition est plutôt marquée par l'atonie : "Une femme, à trois mètres de là, soupira » (Duras, $1958: 7)$. Le « a » final des deux derniers mots semble avoir une valeur conclusive, interdisant tout prolongement sonore à la phrase. La vie d'Anne est, à ce moment-là, aussi fermée et finie que la musique neutre qui l'accompagne. Reportons-nous maintenant à un autre passage du roman où Anne, métamorphosée par la passion, rejoint en pensée l'homme qu'elle désire :

Anne Desbaresdes prend une nouvelle fois son verre qu'on vient de remplir et boit.

Le feu nourrit son ventre de sorcière contrairement aux autres. Ses seins si lourds

de chaque côté de cette fleur si lourde se ressentent de sa maigreur nouvelle et lui font mal. Le vin coule dans sa bouche pleine d'un nom qu'elle ne prononce pas. Cet événement silencieux lui brise les reins. (Duras, 1958 : 109)

La musique est, ici, toute différente. On remarquera les allitérations en /r/ évoquant la brisure des reins et la violence du désir. Parallèlement, le rythme régulier des phrases courtes et affirmatives illustre la détermination du personnage et la forme d'apaisement que lui a apportée la passion. Enfin, au terme du roman, au moment de la séparation des deux "amants" dans un café, on retrouve l'atonie. Anne Desbaredes n'est plus que le sujet de phrases isolées, trop brèves pour faire entendre une autre musique que celle de l'évanouissement et du retour progressif au silence :

Anne Desbaresdes attendit cette minute, puis elle essaya de se relever de sa chaise.

Elle y arriva, se releva. Chauvin regardait ailleurs. Les hommes évitèrent encore de

porter leurs yeux sur cette femme adultère. Elle fut levée. (Duras, $1958: 123$ )

La « mort » d'Anne Desbaresdes est d'abord une mort musicale.

Si, comme on vient de le voir, la musique permet - sur le plan du contenu comme sur celui de l'écriture - de définir l'identité du personnage, c'est également un bon reflet de la façon dont un personnage interagit avec son environnement.

\section{Le vacarme du monde}

Tout récit, si l'on en croit le schéma actantiel de Greimas ${ }^{3}$, peut se concevoir comme une série d'interactions entre personnages. Ces interactions passent, au niveau figuratif, par les actions, les paroles et les pensées. Mais l'essentiel est souvent dans ce qui, sans être explicitement représenté, est simplement suggéré. Or, pour saisir l'implicite et les non-dits des relations entre acteurs, il est souvent intéressant de les considérer sous l'angle du bruit (le son, s'adressant à l'émotion, permet de faire passer des significations qui ne sont pas forcément verbalisables). Une telle analyse n'est pas très difficile à conduire dans la mesure où, dans un récit, la musique d'un personnage que ce soit sur le plan du signifiant ou sur celui du signifié - entre souvent en relation, selon les passages, avec la musique d'autres personnages, avec le bruit de la société, ou avec les sons de la nature. 

des vagues («Le bruit de la mer s'éleva, sans bornes, dans le silence de l'enfant ») (Duras, 1958: 10) s'oppose à la musique sèche, ordonnée et réglée du professeur de piano ( «a dame ponctua cette réponse d'un coup de crayon sur le clavier») (Duras, 1958 : 7). Cette dimension sonore des personnages se retrouve sur le plan du signifiant: l'enfant est souvent associé à une syntaxe déconstruite et ouverte, libérée de la norme et qui laisse entendre un écho sans fin («- Modéré et chantant, dit l'enfant totalement en allé où?») (Duras, 1958: 11), alors que le professeur de piano est associé à la monotonie de la répétition et à la brutalité du ton affirmatif (« - Je ne veux pas savoir s'il est difficile ou non, Madame Desbaresdes, dit la dame. Difficile ou pas, il faut qu'il obéisse, ou bien ») (Duras, 1958 : 9). particulier, le vent («je m'arrêtai avec extase à renifler l'odeur d'un vent coulis qui passait par la porte ») (Proust, 1989 : 335) et la mer (« alors avait commencé le concert symphonique mêlé au clapotement de l'eau, dans lequel les violons vibraient comme un essaim d'abeilles égaré sur la mer ») (Proust, 1989: 176). Si la musique intérieure de Marcel (dont la vibration, comme le montre la citation précédente, est rendue par les allitérations et les assonances) le rapproche de l'univers naturel, elle le coupe souvent de la musique du monde ( je n'appris que le surlendemain, par les journaux, qu'un orchestre tchèque avait joué toute la soirée et que, de minute en minute, s'étaient succédé les feux de Bengale») (Proust, 1989 : 56). Dans la somme proustienne, c'est donc aussi sur le plan sonore que se manifestent la connivence du héros avec la nature et son rapport conflictuel à la mondanité.

Après avoir mis en évidence l'importance de la dimension musicale des personnages, reste à expliquer comment elle conditionne le rapport au texte.

\section{Musique des personnages et programmation de la lecture}

Ce que je voudrais montrer, pour finir, c'est que l'être musical du personnage affecte les différents niveaux de l'acte de lecture.

41 Le plan affectif est le premier à être concerné par la musique. L'identité sonore d'un personnage peut en effet susciter le malaise (sons rugueux, désaccordés, agressifs), l'apaisement (douceur, harmonie), voire la connivence (musique familière) ou l'hostilité (musique culturellement dévalorisée). On ne tranchera pas ici la question de savoir si, au-delà de la dimension culturelle de la musique, il y a une valeur anthropologique des sons. L'essentiel, pour l'analyse littéraire, est de déterminer la valeur de telle musique au sein du système élaboré par chaque texte. Le son strident, par exemple, pourra dans certains récits évoquer l'agression, l'angoisse, la douleur (donc des réalités réputées négatives) alors que dans d'autres il renverra au réveil, au sursaut, à la révolte (donc à des valeurs plutôt positives). Dans L'Emploi du temps de Butor, la ville de Bleston, véritable personnage, fait entendre une musique sourde et hostile. C'est à cette musique négative, relevant du bruit, que Jacques Revel, le hérosnarrateur, tentera d'opposer la musique de l'écriture. Dans K. 622 de Ch. Gailly, la musique de Mozart (« concerto pour clarinette en La majeur»), incarnation sonore de la beauté toujours fuyante, valorise le personnage qui tente de la retrouver. Elle est 
opposée, sur le plan qualitatif, aux Quatre Saisons de Vivaldi, musique qui, devenue l'objet d'une vulgarisation asséchante (elle a envahi les supermarchés comme les standards téléphoniques) connote négativement ceux qui l'apprécient.

L'identité sonore permet aussi de préciser le sens et la fonction d'un personnage. Le système « musical » de chaque texte permet en effet d'identifier sujet, objet, opposant, adjuvant, destinateur et destinataire dans la quête au fondement du récit. Dans $L a$ Recherche, la musique de Vinteuil, reliée à l'intime, au propre et à l'authentique, donc aux valeurs cautionnées par le texte, fait du compositeur un "guide» et, donc, un adjuvant de Marcel. Dans le même ordre d'idées, on évoquera Que ma joie demeure, une nouvelle de M. Tournier qui raconte l'histoire d'un pianiste prodige, Raphaël Bidoche, contraint de gagner sa vie en faisant un numéro de cirque. A la fin de la nouvelle, le vacarme du public, composé de "milliers de bourreaux » aux « rires bestiaux » campe clairement la foule en opposant dans un récit qui fait de l'art musical une valeur. En revanche, la «divine mélodie " (Tournier, 1978: 98) jouée par Bidoche en fait un personnage positif, dernier représentant du sublime dans un monde dégradé.

Enfin, la réalité sonore permet de souligner la dimension symbolique du personnage dans la mesure où elle relie ce dernier aux grandes oppositions qui structurent le champ sémantique humain (nature/culture, vie/mort, humain/non-humain, etc.). La «musique » du personnage nous renverra en effet, selon qu'elle se présente sous une forme élaborée ou qu'elle se réduit à un cri ou un gémissement, plutôt du côté de l'art (culture) ou plutôt du côté des sons primitifs (nature). Les allitérations et le rythme pourront renvoyer à la vitalité, à l'énergie, au dynamisme (c'est-à-dire à la vie) ou, au contraire, à l'amenuisement, l'étouffement, la disparition (la mort). La sonorité pourra être du côté de la voix, de la parole, des sons articulés (donc, de l'humain) ou, au contraire, du côté de l'animalité et de la vie sauvage (le non-humain). On s'intéressera, de ce point de vue, à l'opposition entre Robinson et Vendredi chez Tournier. Alors que Robinson est placé sous le signe de la musique récitative des versets de la Bible, livrephare de la civilisation occidentale, Vendredi ne résonne que d'un rire spontané, archaïque et presque animal. Robinson endosse en effet le rôle de pasteur :

Debout devant le lutrin, il psalmodie les versets de la Bible. Cette lecture est coupée de longs silences méditatifs que suivent des commentaires inspirés par l'Esprit Saint. (Tournier, $1972: 150$ )

Le « sauvage » s'exprime, lui, de façon beaucoup plus rudimentaire :

(...) il rit, il éclate d'un rire redoutable, un rire qui démasque et confond le sérieux menteur dont se parent le gouverneur et son île administrée. Robinson hait ces explosions juvéniles qui sapent son ordre et ruinent son autorité. (Tournier, 1972 : 149)

Significativement, c'est la musique rudimentaire du sauvage qui l'emporte sur la musique construite du civilisé. Dans la suite du récit, Robinson finit, lui aussi, par rire, montrant par là qu'il laisse peu à peu renaître en lui la dimension naturelle de son être.

Dans un tout autre registre, on opposera, dans $W$ ou le souvenir d'enfance de Perec, le rythme inhumain de l'île à la musique humaine du portrait biographique. Voici un passage concernant la description de $\mathrm{W}$, cette étrange cité régie par l'idéal olympique :

Des championnats de classement sortent 264 noms, 66 par village, correspondant aux trois premiers dans chacune des 22 disciplines pratiquées. Les quatre championnats locaux en fournissent quatre fois 66 autres, c'est-à-dire encore 264 ; les deux épreuves de sélection en redonnent deux fois 66, c'est-à-dire 132 (...). (Perec, 1975 : 135) 

objective qui l'énonce, donnent à l'extrait un côté rébarbatif et a-musical, presque cacophonique. Que l'on compare avec ce passage où le narrateur, réfléchissant sur la finalité du récit autobiographique qu'il entreprend, en vient, à travers un style expressif et travaillé, à évoquer ses parents :

J'écris : j'écris parce que nous avons vécu ensemble, parce que j'ai été un parmi eux, ombre au milieu de leurs ombres, corps près de leur corps ; j'écris parce qu'ils ont laissé en moi leur marque indélébile et que la trace en est l'écriture : leur souvenir est mort à l'écriture ; l'écriture est le souvenir de leur mort et l'affirmation de ma vie. (Perec, 1975 : 64)

C'est, ici, la musique, douce et grave à la fois, de la simple humanité qui résonne dans le rythme affirmatif et cadencé de l'écriture.

Pour peu qu'on l'appréhende en termes de bruit, l'être romanesque a donc bel et bien un volume. L'écho du personnage, perceptible à différents niveaux, structure le rapport au texte. Il se laisse même entendre, le livre refermé : ne dit-on pas en effet - et l'on excusera le jeu de mots - que les grands personnages de roman « résonnent » en nous longtemps après la lecture?

\section{BIBLIOGRAPHIE}

ARAGON, Louis (1966). Aurélien. Paris : Gallimard/Folio.

BAKHTINE, Mikhaïl (1970). L'CEuvre de François Rabelais et la culture populaire au Moyen-Age et sous la Renaissance. Paris : Gallimard.

COHEN, Alain (1958). Solal. Paris : Gallimard/Folio.

DURAS, Marguerite (1958). Moderato cantabile. Paris : Minuit.

DURAS, Marguerite (1966). Le Vice-consul. Paris : Gallimard/L'imaginaire.

GAILLY, Christian (1989). K. 622. Paris : Minuit.

GRACQ, Julien (1951). Le Rivage des Syrtes. Paris : Corti.

GREIMAS, Aglirdas Julien (1986). Sémantique structurale. Paris : Presses Universitaires de France.

HUGO, Victor (1967). Les Misérables. I, Paris : Gallimard/Folio.

JAKOBSON, Roman (1963). Essais de linguistique générale. Paris : Minuit.

KUNDERA, Milan (1995). La Lenteur. Paris : Gallimard/Folio.

MALRAUX, André (1946). La Condition humaine. Paris : Gallimard/Folio.

MAUPASSANT (DE), Guy (1974). Toine, in Contes et nouvelles. Paris : Gallimard/La Pléiade.

PEREC, Georges (1975). W. Paris : Gallimard/Denoël.

PICARD, Michel (1989). Lire le temps. Paris : Minuit. 
PROUST, Marcel (1989). Sodome et Gomorrhe. Paris : Gallimard/Folio.

TOURNIER, Michel (1972). Vendredi ou les limbes du Pacifique. Paris : Gallimard/Folio.

TOURNIER, Michel (1978). Que ma joie demeure, in Le Coq de bruyère. Paris : Gallimard/Folio.

VIAN, Boris (1996). L'Ecume des jours. Paris : Pauvert/Le Livre de poche.

\section{NOTES}

1. Cf. Picard (1989: 133-139).

2. Cf. Bakhtine (1970).

3. Cf Greimas (1986).

\section{RÉSUMÉS}

De même que, dans un film, l'arrivée d'un personnage est souvent accompagnée de musique, les figures d'un texte littéraire sont souvent liées à un entourage sonore. Etudier cette "musique » du personnage permet d'appréhender sa valeur, son sens et sa fonction dans un texte donné. Concrètement, comment procéder? A posteriori, quatre champs semblent devoir être retenus. Les deux premiers, le bruit du corps et le rapport à la musique, concernent le plan du contenu ; les deux derniers, le recours aux métaphores musicales et le rythme du texte, sont à étudier au niveau de l'écriture. On montrera, dans un dernier temps, comment l'être musical du personnage affecte les différentes dimensions de l'acte de lecture.

Just as, in a film, the arrival of a character is often accompanied by music, the figures of a literary text are often linked to surrounding sounds. Studying this "music" of the character allows us to understand its value, its meaning and its function in a given text. How to proceed, concretely? A posteriori, four domains should be retained. The first two, the body noise and the correlation to music, pertain to the level of content; the last two, the use of musical metaphors and the rhythm of the text, are to be studied at the level of writing. Finally, we will show how the character's musical being affects the different dimensions of the act of reading.

\section{INDEX}

Keywords : character, music, reading, novel, poetics

Mots-clés : personnage, musique, lecture, roman, poétique

\section{AUTEUR}

\section{VINCENT JOUVE}

Université de Reims

vincent.jouve[at]univ-reims.fr 